\title{
Imaging protein fibers at the nanoscale and in situ
}

\author{
Angelo Bella, Michael Shaw, Emiliana De Santis and Maxim G. Ryadnov
}

\author{
National Physical Laboratory, Teddington, Middlesex, TW11 OLW \\ e-mail: max.ryadnov@npl.co.uk; phone: +44 (0)2089436078
}

\section{Summary}

Protein self-assembly offers a rich repertoire of tools and technologies. However, despite significant progress in this area, a deterministic measure of the phenomenon, which might lead to predictable relationships between protein components, assembly mechanisms and ultimately function, is lacking. Often the challenge relates to the choice of the most informative and precise measurements that can link the chemistry of the building blocks with the resulting assembly, ideally in situ and in real time. Using the example of protein fibrillogenesis - a self-assembly process fundamental to nearly every aspect of biological organisation, from viral assembly to tissue restoration - this chapter demonstrates how protein self-assembly can be visually and precisely measured while providing measurement protocols applicable to other self-assembly systems.

Key Words: molecular self-assembly, nanometrology, real-time imaging, super-resolution microscopy, nanoscale biophysics, protein fibrillogenesis

\section{Introduction}

The ability to construct biological matter from the molecule up holds promise for technologies that are set to shape our future (1). Synthetic biology, regenerative medicine and advanced materials are just a few areas where engineered self-assembling systems can contribute effective solutions (2). Such solutions are not limited to commercial adaptations, and can be insightful to advance the mechanistic understanding of biomolecular assembly (3). From infection to tissue restoration, self-assembly mechanisms underpin a variety of biological processes by encoding the hierarchical organization of predominantly protein molecules into the complex architectures of viruses, cells and tissues.

There exist different forms and shapes of self-assembled systems. Most of them can be grouped into the generic units of particles (viruses) and tubes or fibers (microtubules, collagen fibres) (4). These units alone can support specialist functions or can arrange into higher-order functional systems (extracellular matrices, cells). Arguably, however, protein fibers present the richest repertoire of functional assemblies that are involved in both normal and pathogenic processes.

Indeed, diverse information including, but not limited to, cell proliferation and tissue development as well as neurodegeneration and viral assembly is realized through protein fibrillogenesis (5-9). Resulting structures are near crystalline (10), which implies a nanoscale order, and grow hierarchically (11), which implies their assembly is polar and directional (12). To understand the process and link it with the assembled structures requires suitable fibrillogenesis models that would be free of other events and modifications, which are not necessarily involved in fiber formation. De novo amino-acid sequences based on archetypal folding motifs provide a straightforward solution (13). This so-called reductionist approach allows distinguishing folding-assembly pathways from unrelated events thereby helping to reveal fibre formation as a cooperative process at the nanoscale. To achieve this, high-resolution and real-time imaging approaches are necessary.

Since this chapter is a practical guide to the imaging of protein fibers, it starts with basic design principles to enable protein fibrillogenesis, for which we focus on helical assemblies. It then describes microscopy methods, traditional and innovative, which can be used for the visual characterisation of fiber formation in situ and in real time. 




Cytoskeleton

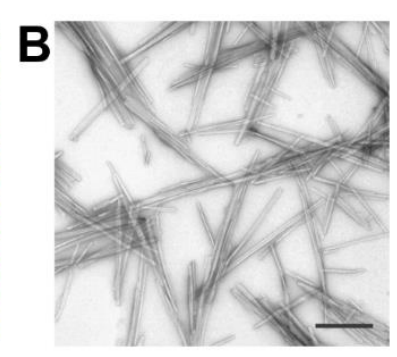

Amyloid fibril



Filamentous virus

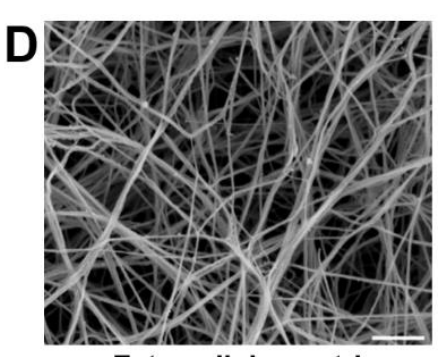

Extracellular matrix

Figure 1. Examples of naturally occurring protein fibers. (A) Actin fibers (green) forming cytoskeleton with nuclear DNA highlighted in blue (14). (B) Transthyretin-derived amyloid fibrils (scale bar $200 \mathrm{~nm}$ ) (15). (C) Tobacco mosaic virus (scale bar $100 \mathrm{~nm}$ ) (16). (D) Type I collagen extracellular matrix (scale bar $1 \mu \mathrm{m}$ ) (17). Reproduced: by permission of the Royal society of chemistry (A and C), from ref (15), copyright (2002) National Academy of Sciences, USA (B) and by permission of the Journal of Cell Science by Company of Biologists Ltd (D).

\subsection{Basic principles for designing protein fibers}

Naturally occurring fibers use monomeric subunits whose amino-acid sequences support particular oligomerisation states and propagation modes. Longitudinal assembly appears to dominate and often occurs at the expense of complementary subunits placed axially out of register. Examples may include $\alpha$-helical intermediate filaments assembled from staggered monomer (20). $\beta$-pleated amyloid fibrils that grow from out-of-register $\beta$-sheets (21) or collagen fibers consisting of tropocollagen molecules held together side by side in an offset fashion (22). 

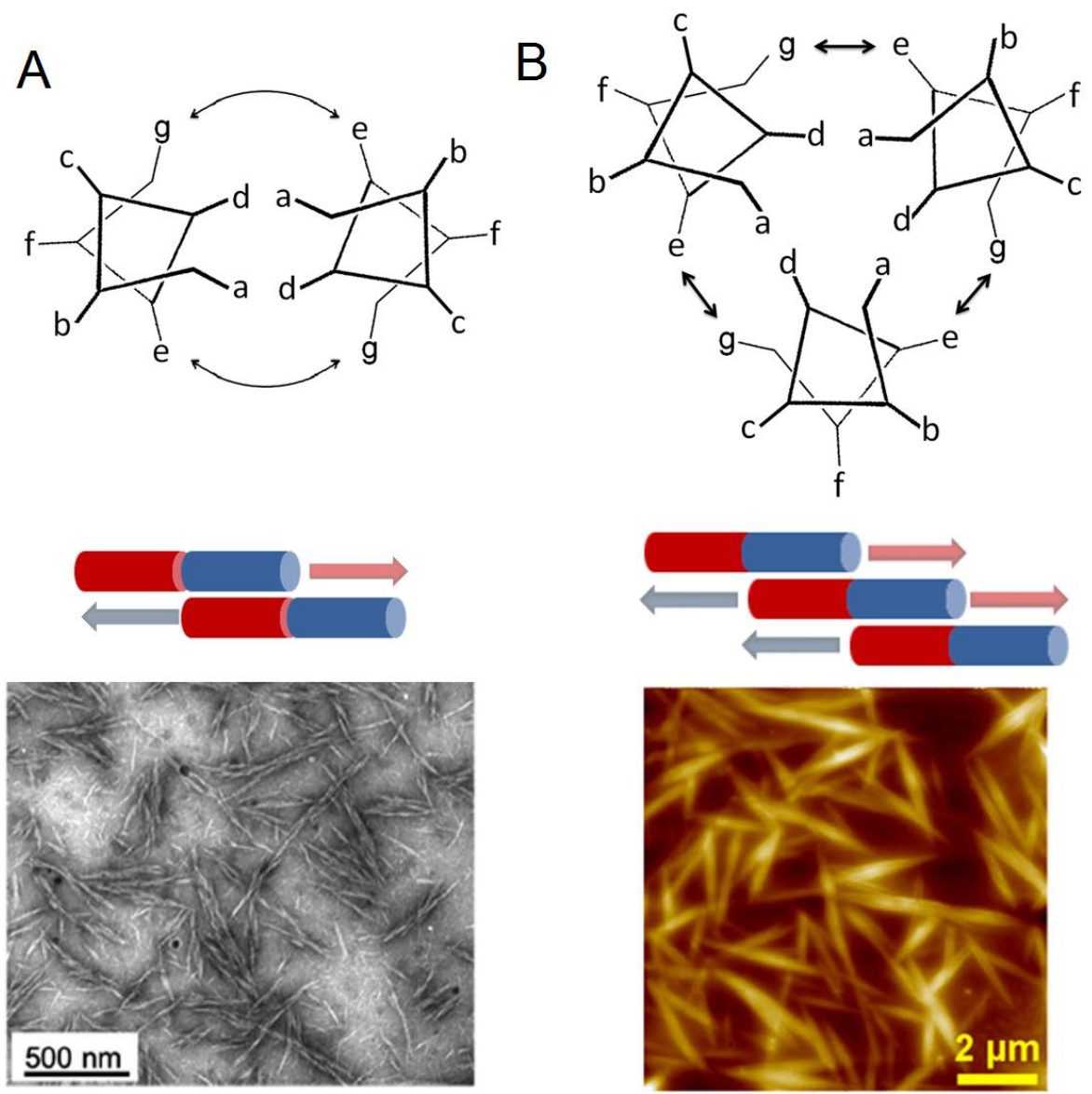

C
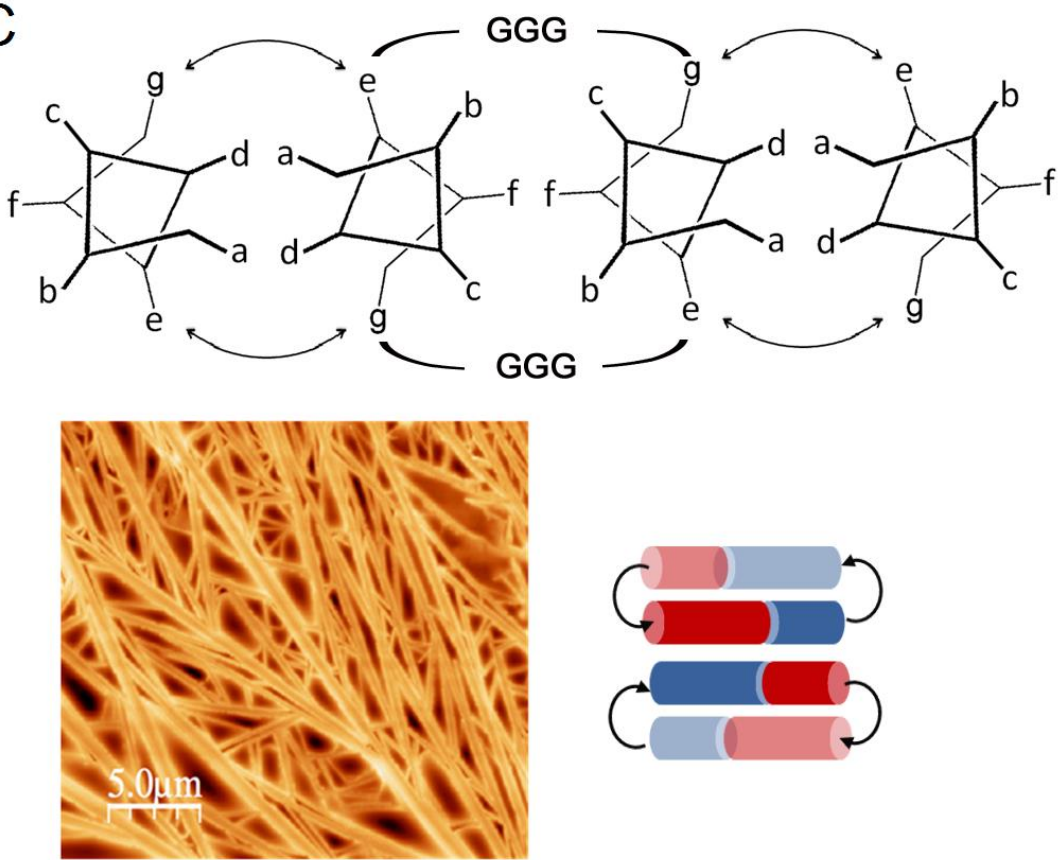

Figure 2. Coiled-coil heptad repeats, gabcdef, configured into helical wheels for dimeric (T2, A) (14), trimeric (FiM, B) (23) and dimeric cyclised (Cycl_one, C) (24) self-assembling subunits (upper) together with the resulting fibrillar structures (lower). Reproduced by permission of the Royal society of chemistry (A), Adapted by permission from Macmillan Publishers Ltd: Scientific Reports, (23) Copyright 2014 (B) and Angew Chem Intl Ed, John Wiley and Sons (24) (C). 
Over the last decade a number of de novo designs based on $\alpha$-helical coiled-coil motifs applied the same principles of staggered assembly. Coiled coils are rope-like bundles of two or more helices that interdigitate into a super-helix. The sequences of coiled coils show an amphipathic heptad repeat of polar (P) and hydrophobic $(\mathrm{H})$ residues, namely PHPPHPP (25). The repeat is often designated gabcdef and depicted schematically using coiled-coil helical wheels with 3.5 residues per turn (as opposed to 3.6 residues per turn for non-oligomerising helices). A clear advantage of coiled coils is that the heptad repeat, which spans $1 \mathrm{~nm}$, can be used as a construction module (26) and when combined with staggered assembly, the sequences comprising as few as 4 heptads, each giving two two-heptad overhangs, can assemble into micron-long fibers (Figure 2) (13) (23).

To ensure complementary sequence offsets, designed coiled coils use charge-charge interactions at $g$ and $e$ sites of successive heptads ( $g-e^{\prime}$ interactions) (27). Specifically, each of two generic heptads that use cationic (lysines) and anionic (glutamates) residues can be paired sequentially to provide the oppositely charged sticky ends (10). The choice of hydrophobic residues in $a$ and $d$ defines the oligomerisation state of the self-assembling subunit (Figure 2 ) including fiber-forming sticky-ended dimers (10) (14), trimers (23) (28) and pentamers (29).

The other sites of the heptad unit ( $b, c$ and $f$ ) are usually polar (glutamines) or small (alanines) amino acids having high helical propensities. Single aromatic residues (tryptophans, tyrosines) in $f$ are often used as chromophores for accurate concentration measurements. Additionally, to fine-tune interstagger interactions tryptophans can be regularly spaced at super-helix pitch distances $(1.63 \mathrm{~nm})$ thereby promoting the zippering up of coiled-coil ropes into discrete filaments while arresting fiber thickening (Figure 3) (30).

\subsection{Morphology on demand}

In contrast to $\beta$-structured and collagen-like fibers, $\alpha$-helical designs allow for a better control and substantial orthogonality over the assembly. This property has been successfully used to rationally engineer nanoscale order (10) and nano-to-microscale morphologies into resulting fibrous materials including kinked (31) branched (32) and network-like (24) (33) architectures (Figure 2). The head-totail cyclisation of coiled-coil sequences was shown to guide the arbitrary propagation of selfassembling subunits resulting in matrices and nets that can span unprecedented nano-to-submillimetre dimensions (24) (33). Unlike fibers derived from other folding types, helical designs are strongly prone to thickening by maturation. Yet, by carefully tuning interfacial coiled-coil contacts it is possible to limit the lateral association of coiled-coil bundles (30). Moreover, the length and thickness of resulting fibers can be empirically correlated with the length of designed peptide sequences (14).

\subsection{Material requirements for imaging}

Protein fibers exhibit nanoscale order which can be engineered rationally (10). The accurate characterisation of the assembled structures requires high resolution methods. These provide endpoint measurements that do not depend on the knowledge of intermediate steps in fiber formation or growth kinetics. X-ray fiber diffractions (XRD) from oriented and partly dried fiber assemblies are obtained to gain detailed insights into fiber ultrastructure (34). XRD data is used to complement the visual evidence of fiber assembly providing structural correlations with fiber morphology. Transmission electron microscopy (TEM) is the most commonly used visualisation technique in this regard. 

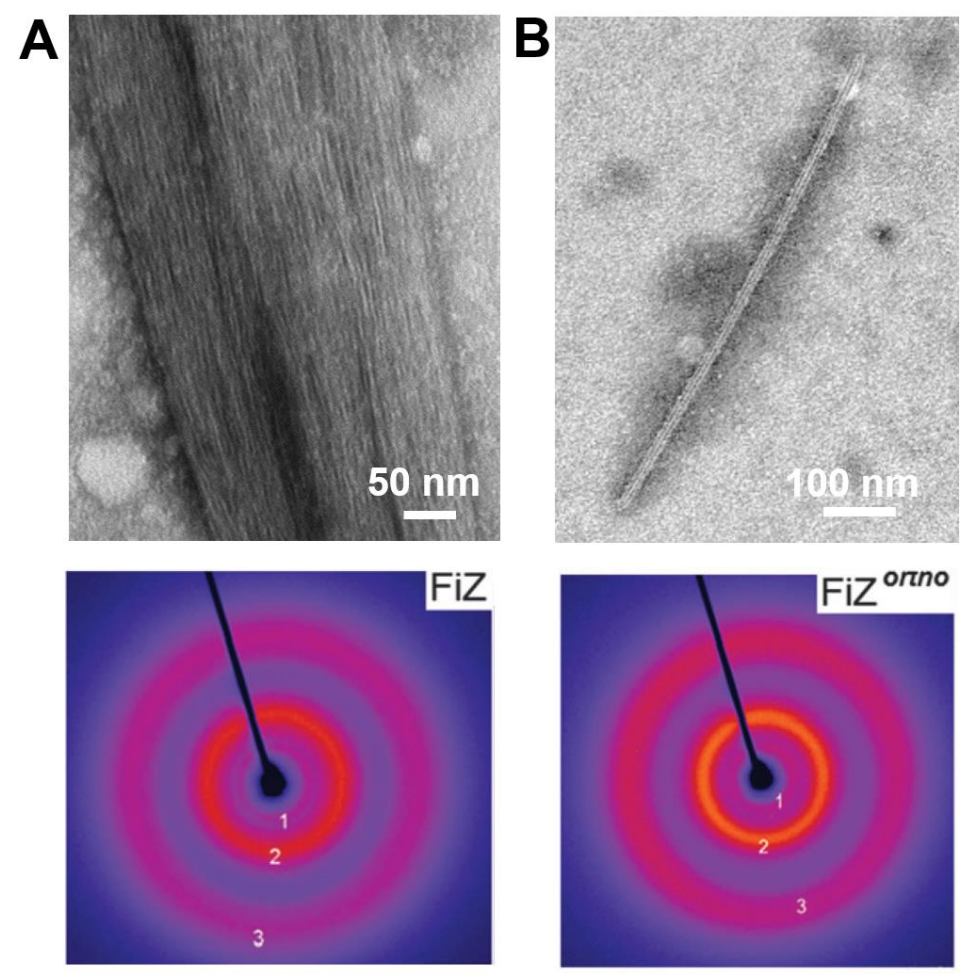

Figure 3. Zippering up of coiled coil ropes into individual filaments via interfacial aromatic interactions. (A) Transmission electron micrographs (top) and X-ray diffraction patterns (bottom) for a filamentous coiled-coil zipper ( $F i Z, A$ ), which gives individual filaments bundled together without maturation, and its orthogonal version (FiZ $\left.{ }^{\text {ortho }}, \mathrm{B}\right)$, whose filaments are less prone to bundling up. Reproduced from (30) with permission from the PCCP Owner Societies.

Figure 3 gives an example of correlations between coiled-coil structure, packing and assembly. The XRD patterns for the two designs of coiled coil fibers show typical intra-helical distances for discrete coiled coils and close packing distances between coiled coils in assembled filaments. The pattern for the orthogonal design matches closely the size of 3D hexagonal lattices for axially packed coiled-coil staggers, which confirms super-helix contiguity despite the orthogonal (via a side chain) linkage of two two-heptad overhangs. TEM confirm the filamentous assembly of the coiled-coil staggers with consistent differences in the bundling of individual filaments (30).

TEM provides a nanometre spatial resolution, but does not allow for trade-offs with temporal measurements. Cryogenic TEM coupled with tomography offers a somewhat more advanced approach in gaining more precise and less biased structural information that is free of sample staining and drying. However, self-assembly is a living mechanistic process, the complete understanding of which demands imaging capabilities with sufficient temporal resolution to monitor fiber growth (12) (22). Optical microscopy methods are minimally invasive and well suited for imaging live processes under ambient conditions, but are limited in spatial resolution ( $200 \mathrm{~nm})$, which often prevents from detecting small structural changes. Super-resolution fluorescence imaging improves spatial resolution beyond the diffraction limit (approximately half the wavelength of light used for imaging), with structured illumination microscopy (SIM) being of particular interest for the present application owing to its fast image acquisition rate (35), low sample light exposure, compatibility with conventional fluorescent labelling methods. SIM works by illuminating the sample with spatially patterned light, causing previously undetectable high spatial frequency image information to be visible as low frequency moiré fringes (36). By capturing a series of images under different illumination patterns this high frequency information can be shifted back to its origin in Fourier space allowing reconstruction of a 'super-resolved' image with lateral spatial resolution down to $100 \mathrm{~nm}$ (37). Such images can be used to track microscopic self-assembly in real time, however for further insights in the process which 
can be gained through even higher spatial resolution it is necessary to consider alternative methods. Atomic force microscopy (AFM), in particular, can be performed in water and does not require the use of labels to generate image contrast. However, AFM measurements are position- and surfacedependent and subject to sample drifting. A compromise therefore is often proposed in materials themselves.

For example, to delineate fibrillogenesis as a mechanistic process, homogeneous assembly models are crucial. Naturally occurring structures, taken out of their native context, are not suitable for the purpose. Large heterogeneities can be observed in reconstituted fiber morphologies (extracellular matrices) or in growth rates derived from inherent structural polymorphism (amyloids) (38). In contrast, archetypal designs based on simplified sequence templates (coiled-coil heptads) and generic assembly modes (staggers) can give relatively uniform fiber structures of statistically significant densities to allow for the analysis of growth kinetics (23). Peptide designs can readily accommodate orthogonal modifications to incorporate fluorophores (i.e. $f$ positions in coiled coils) thereby supporting in-situ and real-time imaging of fiber formation. More complex fibrous assemblies including branching matrices and knotted networks are far less predictable. Their designs can only be partly satisfied by deliberately introduced promiscuity in fiber formation, which provides a level of control over resulting structures (networked as opposed to straight fibers). All in all, the SIM imaging of an archetypal protein fiber appears as an obvious solution to the real-time measurements of fiber growth kinetics.

\section{Materials}

\subsection{Peptide synthesis}

\subsubsection{9-Fluorenylmethoxycarbonyl (Fmoc) Solid Phase Peptide Synthesis}

1. Solvents: Dimethylformamide (DMF), N,N-diisopropylethylamine (DIPEA), dichloromethane (DCM) trifluoroacetic acid (TFA) tri-isopropyl silane (TIS).

2. Fmoc-amino acids: Fmoc-Ala-OH, Fmoc-Gln(Trt)-OH, Fmoc-Glu-(Ot'Bu)-OH, Fmoc-Glu-OAll, Fmoc-lle-OH, Fmoc-Lys(Boc)-OH, Fmoc-Leu-OH, Fmoc-Lys(Aloc)-OH, Fmoc-Tyr('Bu).

3. Activating agent: O-benzotriazole- $N, N, N^{\prime}, N^{\prime}$-tetramethyluronium hexafluorophosphate (HBTU).

4. Resins: (100-200 mesh 0.36-0.67 mmol/g) Fmoc-Gln(Trt)-Wang resin or Rink Amide MBHA.

5. Fmoc deprotection: $20 \%$ piperidine in DMF.

6. Allyl deprotection: Tetrakis(triphenylphosphine)palladium(0) (4 eq) in $\mathrm{CHCl}_{3} / \mathrm{AcOH} \mathrm{DIPEA}$ 3.6/0.2/0.2 mL.

7. Coupling mixture: $0.5 \mathrm{M}$ HBTU and $1 \mathrm{M}$ DIPEA in DMF.

8. Cleavage cocktail: TFA/TIS/water (95:2.5:2.5).

\subsubsection{Peptide Labelling}

1. Alexa Fluor 488- carboxylic acid, succinimidyl ester.

2. DIPEA (2eq) in DMF.

\subsubsection{Reversed Phase High-Performance Liquid Chromatography}

1. Mobile phases: $5 \%$ acetonitrile $(A c N)+95 \%$ water (buffer A), $5 \%$ Water $+95 \%$ AcN (buffer B) both containing $0.1 \%$ TFA. All solvents are HPLC grade.

2. Stationary phase: Vydac $\mathrm{C} 8$ and $\mathrm{C} 18$ reverse-phase columns. Analytical $(5 \mu \mathrm{m}, 4.6 \mathrm{~mm}$ i.d. $\mathrm{x}$ $250 \mathrm{~mm}$ ) and semi-preparative ( $5 \mu \mathrm{m}, 10 \mathrm{~mm}$ i.d. $\times 250 \mathrm{~mm}$ ).

3. High Performance Liquid Chromatographic (HPLC) system (Jasco-2080 with UV 2072 detector). 


\subsubsection{Mass Spectrometry}

1. MALDI ToF (Bruker Daltonics) Autoflex III smartbeam, with flex control as proprietary software.

2. 2,5-Dihydroxybenzoic acid or sinapinic acid as matrix.

\subsection{Fiber Assembly}

Peptides described in table 1 below are incubated in MOPS (10 mM, pH 7.4) overnight prior to endpoint imaging (TEM, AFM). All solutions use filtered $(0.22 \mu \mathrm{m})$ water with a resistivity of $18.2 \mathrm{M} \Omega$.

Table 1 Sequence template with varied integral module numbers

\begin{tabular}{cl} 
Name $^{\mathrm{a}}$ & \multicolumn{1}{c}{ Sequence } \\
\hline FiZ & KLAALKWKLAALKQKLAALKWELAALEQELAALEWELAALEQ \\
Fiz ${ }^{\text {rtho }}$ & KLAALKWKLAALKQKLAALKX (ELAALEQELAALEWELAALEQ) ${ }^{\text {b }}$ \\
FiM & KLAALKQKLAALKKELAALEQELAALEQ \\
FiM(Alexa) & KLAALKQKLAALKK (Alexa4 88) ELAALEQELAALEQ \\
T2 & KIAALKQKIAALKKEIAALEYEIAALEQ \\
CYcl_one & CYClo(KIAALKYEIAALEKIAALKGGGEIAALEQKIAALKQEIAALEGGG)
\end{tabular}

${ }^{\text {aOriginal names; }}{ }^{b} \mathrm{X}$ is a glutamate forming an isopeptide (orthogonal) bond with the first glutamate of the anionic domain

\subsection{Spectroscopy}

\subsubsection{Circular Dichroism}

1. Quartz cuvettes (0.1 and $0.05 \mathrm{~cm}$ from Starna UK).

2. Chirascan Plus spectropolarimeter (Applied Photophysics Ltd.) equipped with a Peltier temperature controller.

2.3.2. Linear Dichroism

1. Micro volume quartz cuvette flow cell with $0.5 \mathrm{~mm}$ annular gap and quartz capillaries (Kromatec Ltd, UK).

2. Jasco 810 spectropolarimeter using a photoelastic modulator $1 / 2$ wave.

\subsubsection{Fourier-transform Infrared (FT-IR)}

1. Tensor-37 series FTIR spectrophotometer with a BioATR II unit (Bruker Optics) with photovoltaic MCT detector and a Bruker optics workstation equipped with OPUS software.

\subsection{Microscopy}

\subsubsection{Structured Illumination Microscopy}

1. Custom-built structured illumination microscope (18-19) based on an inverted microscope body (IX71, Olympus) with high numerical aperture (UAPON 100XOTIRF, Olympus) and silicone immersion (UPLSAPO 60XS, Olympus) objective lenses for total internal reflection fluorescence (TIRF) and wide field epifluorescence imaging, respectively. Images are captured with a scientific CMOS camera (Flash 4.0 v1, Hamamatsu Photonics).

2. Time-lapse imaging: Performed in TIRF mode using a 100X/1.49 objective lens with a typical exposure time of $100 \mathrm{~ms}$. Super-resolution images of the assembled fibers are captured using a $60 x / 1.3$ objective lens. 
3. Sample mounting: samples are mounted in a high precision z-stage (NanoScan, Prior Scientific Ltd.) in closed loop with a focus stabilisation system (CRISP, Applied Scientific Instrumentation). Illumination patterns generated using a spatial light modulator (SXGA3DM, Forth Dimension Displays) illuminated using a $488 \mathrm{~nm}$ laser (Sapphire, Coherent Inc.). Additional optics and optomechanics are from Thorlabs and Qioptiq. All hardware is controlled using custom software that is written in LabVIEW 2013 (National Instruments). Software can be tailored to specific hardware requirements for bespoke microscopes or can be proprietary for commercial instruments

4. Super-resolution image reconstruction: custom software written in Matlab (MathWorks) as detailed elsewhere $(18,19)$.

5. Filament segmentation: ImageJ software.

6. Sample chambers: chambered cover glass with 8 wells (NUNC Lab-TEK II, Thermo Scientific).

\subsubsection{Transmission Electron Microscopy}

1. Support grid: formvar/carbon coated copper/palladium support grids or copper grids coated with pioloform and carbon film. All subjected to plasma glow discharge before use.

2. Staining reagent: $2 \%$ phosphotungstic acid or $1 \%$ uranyl acetate in distilled water filtered through a $0.2 \mu \mathrm{m}$ pore size Minisart unit.

3. Microscope: Philips BioTwin transmission electron microscopy with $80 \mathrm{kV}$ accelerating voltage or FEI Tecnai 20 with $200 \mathrm{kV}$ accelerating voltage.

4. Image analysis: ImageJ software.

\subsubsection{Cryo-Transmission Electron Microscopy}

1. Support grid: lacey carbon grids (EM Resolutions Ltd.) subjected to plasma glow discharge before use.

2. Sample freezing: plunge freezing into liquid ethane cooled with liquid $\mathrm{N}_{2}$ and performed using a VITROBOT mark IV (FEI Company).

3. Microscope: FEI Tecnai 20 with $200 \mathrm{kV}$ accelerating voltage and fitted with an Eagle $4 \mathrm{k} \times 4 \mathrm{k}$ camera (FEI).

4. Image analysis: ImageJ software.

\subsubsection{Atomic force microscopy}

1. Clean silicon wafer $7.0 \mathrm{~mm} \times 5.0 \mathrm{~mm}$ with $100 \mu \mathrm{m}$ diameter and $525 \mu \mathrm{m}$ thickness.

2. Cypher AFM instrument (Asylum research).

3. AFM tips: super sharp silicon probes (nanosensors) with resonant frequency of $\sim 330 \mathrm{kHz} .5$ $\mathrm{nm}$ radius of curvature, force constant $42 \mathrm{~N} / \mathrm{m}$.

4. Proprietary imaging software SPIP 6.0.2.

\subsection{Fiber diffraction of partially aligned samples}

\subsubsection{Partial alignment of fibers: stretched frame procedure}

1. Borosilicate thin-walled capillaries (1.5-mm outer dimeter, 1.17-mm inner diameter) (Harvard Apparatus).

2. Cutting stone (Hampton Research).

3. Standard beeswax: break into pieces, transfer the pieces into a glass beaker.

4. Mounting clay (Hampton research)

5. Petri dishes, 90-mm diameter 
6. Parafilm ${ }^{\circledR}$

\subsubsection{Fiber diffraction}

1. Rigaku CuKa rotating anode X-ray source (wavelength $1.5418 \AA$ ) and R-AXIS IV++ diffractometer

2. Instrument control and data collection through the CrystalClearTM software (Rigaku).

3. Data handled and converted to image files in Tiff formats.

\section{Methods}

\subsection{Synthesis of model fiber-forming subunits}

Model peptide sequences are relatively short and straightforward for chemical synthesis. These are assembled on a solid support (polymer resin beads) using fully automated peptide synthesisers. Sitespecific modifications including fluorescent labelling, branching and cyclisation can be performed manually on the same substrate. After the modifications are complete, which can be confirmed by mass spectrometry, the assembly of the sequence can continue till completion, if necessary, and the synthesized product is then cleaved from the support for post-synthetic purification and analysis.

\subsubsection{Peptide Synthesis}

Solid-phase peptide synthesis is performed using standard $\mathrm{Fmoc} /{ }^{t} \mathrm{Bu}$ protocols that allow significant flexibility regarding coupling efficiencies, synthesis scale and time, solvent and reagent use and ultimately product yields. The same protocols were used for the incorporation of orthogonal protecting groups (allyl for Cycl_one, Mtt for Fizortho) to allow site-specific side-chain modifications. Uronium salts (HBTU, HCTU) together with DIPEA were used as coupling reagents to assemble the sequences reported in Table 1.

3.1.1.1. Perform peptide synthesis at a $100 \mu \mathrm{M}$ scale on a MBHA rink amide resin (100-200 mesh). 3.1.1.2. Use an automated peptide synthesiser (CEM Liberty) according to the following synthesis cycle:

1. Deprotect Fmoc protected resin/amino acids by $20 \%$ piperidine in DMF $(7 \mathrm{~mL})$ for 5 minutes.

2. Wash $(x 4)$ the resin with $\operatorname{DMF}(7 \mathrm{~mL})$.

3. Perform amino-acid coupling (10 $\mathrm{min})$ using Fmoc-protected amino acid ( $2.5 \mathrm{~mL}, 0.2-0.4 \mathrm{M})$ with HBTU or HCTU $(0.5 \mathrm{M}, 1 \mathrm{~mL})$ and DIPEA $(1 \mathrm{~mL}, 1 \mathrm{M})$. All are in DMF.

4. Wash $(x 4)$ the resin with DMF $(7 \mathrm{~mL})$.

3.1.1.3. For orthogonal allyl/Alloc deprotection:

1. Add tetrakis (triphenylphosphine) palladium (0) $(400 \mu \mathrm{M})$ in chloroform/DIPEA/AcOH (36:0.2:0.2) containing phenylsilane (4 eq.) as a scavenger.

2. Incubate for 2-16 hours with gentle agitation in the dark.

3. Wash with $0.5 \%$ sodium diethylthiocarbammate ( $w / v$ in DMF), $0.5 \%$ DIPEA ( $v / v$ in DMF) and DMF.

4. Repeat washing (x3).

3.1.1.4. For post-synthesis cleavage:

1. Per-wash resin with DCM to remove residual DMF.

2. Keep the resin under cleavage cocktail ( $10 \mathrm{~mL} / \mathrm{g}$ of resin) for 3-4 hours.

3. Recover the liquid phase by filtration (main filtrate) and the resin rinsed with another $5 \mathrm{~mL}$ of the cocktail (second filtrate). 
4. Add a three-fold excess of diethyl ether to each filtrate.

5. Centrifuge the filtrates at $3500 \mathrm{rpm}$ for $45 \mathrm{~min}$ at $4^{\circ} \mathrm{C}$.

6. Discharge the supernatant to give peptide precipitates.

\subsubsection{Lyophilisation:}

1. Dissolve the obtained precipitates in distilled water and freeze.

2. Freeze-dry the solidified sample under vacuum.

\subsubsection{Labelling and cyclization}

Side chain modifications (fluorophores - FiM-Alexa), orthogonal conjugation (FiZ ${ }^{\text {ortho }}$ ) and cyclization (Cycle_one) are carried out by applying $\mathrm{Fmoc} /{ }^{\mathrm{B}} \mathrm{Bu} / \mathrm{Mtt}$ or Fmoc/ ${ }^{t} \mathrm{Bu} / \mathrm{Allyl}$ protocols (Table 1). FmocLys(Aloc)-OH, Fmoc-Lys(Mtt)-OH and Fmoc-Glu(OAl)-OH derivatives are used to incorporate modification sites in fully protected sequences directly on resin. Allyl and Mtt chemistries are orthogonal to the $\mathrm{Fmoc} /{ }^{\mathrm{t}} \mathrm{Bu}$ protocols and do not affect other residues.

\subsubsection{Fluorescent labelling of FiM-Alexa:}

1. Mix an equivalent of orthogonally deprotected resin (-Lys(Aloc)-) with Alexa Fluor 488 succinimidyl ester/DIPEA (1.1 eq. in DMF).

2. Perform the reaction at $60^{\circ} \mathrm{C}$ for $10 \mathrm{~min}$ in a microwave unit (CEM Corp.).

\subsubsection{Backbone cyclization:}

1. Load Fmoc-Glu-OAll onto the MBHA resin as the first residue through the $\gamma$-carboxylate.

2. Assemble the sequence using the cycle described in 3.1.1.2 and when complete remove the allyl group using the mixture in 3.1.1.3 which will leave the other residues protected.

3. Once the Alloc group is removed cyclise the peptide directly on the resin using a 4-fold excess of HBTU and DIPEA (total $4 \mathrm{~mL}$ ) at $60^{\circ} \mathrm{C}(10 \mathrm{~min})$.

4. Repeat the cyclization step twice.

5. Cleave the resin and process the product using the protocol described in 3.1.1.4-3.1.1.5. Note: upon cleavage the glutamate is converted into glutamine.

\subsubsection{Reverse Phase-HPLC and mass spectrometry}

1. Solubilise the crude peptide in distilled water.

2. Inject $5 \mathrm{~mL}$ of the obtained peptide solution on a semi-preparative RP-HPLC column (Vydac $\left.\mathrm{C}_{18}, 5 \mu \mathrm{m}\right)$.

3. Run a $10-70 \%$ gradient of $95 \%$ aq. $\mathrm{CH}_{3} \mathrm{CN}$ containing $0.1 \%$ TFA over $50 \mathrm{~min}$, with the flow rate of $4.7 \mathrm{~mL} / \mathrm{min}$.

4. Monitor the gradient at different wavelengths: $214 \mathrm{~nm}$ and $230 \mathrm{~nm}$ (peptide bonds), 280 $\mathrm{nm}$ (aromatic side chains) and $495 \mathrm{~nm}$ (Alexa Fluor 488).

5. Collect eluted fractions every 30 seconds.

6. Mix $1 \mu$ l of DHB matrix ( $10 \mathrm{mg} / \mathrm{mL}$ in $\mathrm{CH}_{3} \mathrm{CN} /$ water $50: 50$ containing $0.1 \%$ TFA) with peptide $(1 \mu \mathrm{l})$ and spot $1 \mu \mathrm{l}$ of the obtained mixture on a MALDI-TOF plate to confirm peptide identity

7. Combine pure fractions, freeze and freeze-dry as per 3.1.1.4-3.1.1.5.

8. Solubilise an aliquot of the purified peptide to confirm and quantify its purity by analytical RP-HPLC (Vydac $\left.\mathrm{C}_{18}, 5 \mu \mathrm{m}\right)$ at the flow rate of $1 \mathrm{~mL} / \mathrm{min}$.

\subsection{Fiber assembly}

\subsubsection{Fiber preparations for TEM imaging}

1. Prepare a stock solution $(0.5-1 \mathrm{~mL})$ of a pure peptide $(2-3 \mathrm{mM})$. 
2. Determinate peptide concentration by UV-Vis for FiM(Alexa) at $495 \mathrm{~nm}\left(\varepsilon\right.$ of $71000 \mathrm{M}^{-1} \mathrm{~cm}^{-}$ ${ }^{1}$ ), for FiM at $214 \mathrm{~nm}$ calculating extinction coefficient as described elsewhere (39) and at $280 \mathrm{~nm}$ for all other peptides (Table 1 ).

3. Prepare peptide samples $(200 \mu \mathrm{L}, 100 \mu \mathrm{M})$ in $10 \mathrm{mM}$ MOPS or $10 \mathrm{mM}$ phosphate buffer, $\mathrm{pH}$ 7.4 .

4. Incubate at room temperature overnight.

\subsubsection{Fiber preparations for optical microscopy}

1. Prepare a $10 \mu \mathrm{M}$ solution of FIM (Alexa) in $10 \mathrm{mM}$ MOPS, pH 7.4.

2. Prepare FiM(Alexa)/FiM samples at a $1: 10^{4}$ molar ratio using the obtained solution and the stock (FiM).

3. Place the resulting solution into a well of an 8-well chamber ( $\mathrm{Nunc}^{\mathrm{TM}}$ Lab-Tek $^{\mathrm{TM}}$ ).

\subsubsection{Transmission electron microscopy (staining)}

1. Deposit $8 \mu \mathrm{L}$ of a fiber preparation (as above) on a glow-discharged TEM grid.

2. Let the content of the droplet settle for $1 \mathrm{~min}$.

3. Remove excess solution carefully by blotting paper from one side of the grid.

4. Alternatively, deposit a TEM grid upside down on a droplet placed on a piece of clean Parafilm ${ }^{\circledR}$.

5. Remove excess water by blotting.

6. Stain the sample grid for $10 \mathrm{sec}$ with uranyl acetate, phosphotungstic acid or ammonium molybdate $(8 \mu \mathrm{L}, 1 \% \mathrm{w} / \mathrm{v})$.

7. Remove excess stain by blotting, and transfer the grid into a TEM microscope for imaging.

3.2.3 Cryo-transmission electron microscopy (no staining)

1. Prepare samples in a climate chamber with $100 \%$ relative humidity.

2. Load a droplet $(5 \mu \mathrm{L})$ of a peptide solution on a glow discharged lacey carbon grid.

3. Let the content of the droplet settle for 2-10 s before blotting.

4. Plunge into liquid ethane cooled with liquid $\mathrm{N}_{2}$ for freezing. Peptide nanostructures become embedded in a vitreous ice suspended inside the holes of the carbon grid.

5. Transfer the sample grid (without warming) into a Gatan 626 cryo-holder filled with liquid $\mathrm{N}_{2}$ and image.

\subsection{Spectroscopy}

\subsubsection{Circular Dichroism spectroscopy}

\subsubsection{CD spectra}

1. Prepare peptide sample $(50-300 \mu \mathrm{M})$ in $10 \mathrm{mM}$ MOPS or phosphate buffer, $\mathrm{pH} 7.4$

2. Place the sample in a quartz cuvette with 0.05 or $0.1 \mathrm{~cm}$ path length

3. Record far-UV CD spectra in the $190-260 \mathrm{~nm}$ wavelength range collecting data points at 1 $\mathrm{nm}$ step, $1 \mathrm{~nm}$ bandwidth with 4 acquisitions

4. Convert all the measurements, after baseline correction, into mean residue ellipticities by normalising for the concentration of peptide bonds and cuvette path length (MRE, deg $\mathrm{cm}^{2}$ $\mathrm{dmol}^{-1} \mathrm{res}^{-1}$ ).

\subsubsection{Thermal denaturation curves}

1. Record unfolding curves at $222 \mathrm{~nm}$ in the range of $5^{\circ} \mathrm{C}$ to $90^{\circ} \mathrm{C}$ at $1^{\circ} \mathrm{C}$ intervals with a ramp of $1^{\circ} \mathrm{C} / \mathrm{min}$ and 16 seconds equilibration time for each temperature point.

2. Record CD spectra in the $190-260 \mathrm{~nm}$ wavelength range after the melt and cooling.

3. Plot the thermal unfolding curves as a function of temperature in mean residue ellipticities.

\subsubsection{Linear Dichroism Spectroscopy}

1. Place a peptide aliquot ( $60 \mu \mathrm{L}, 100 \mu \mathrm{M}$ in $10 \mathrm{mM}$ MOPS, pH 7.4) into a micro-volume quartz Couette flow cell. 
2. Achieve molecular alignment by setting up a constant laminar flow of the sample solution between the two coaxial cylinders, a stationary quartz rod and a cylindrical capillary rotating at $3000 \mathrm{rpm}$.

3. Record all spectra in the $260-190 \mathrm{~nm}$ wavelength range at a $1 \mathrm{~nm}$ step size, $1 \mathrm{~nm}$ bandwidth, $50 \mathrm{~nm} / \mathrm{min}$ scan speed, with a 4-sec response time and 3 acquisitions.

4. Process the spectra by subtracting non-rotating baseline spectra and normalising for the concentration of peptide bonds with conversion to a mean residue delta epsilon $\Delta \varepsilon\left(\mathrm{M}^{-1} \mathrm{~cm}^{-}\right.$ ${ }^{1}$ res $\left.^{-1}\right)$.

3.3.3 Fourier Transform Infra-Red spectroscopy

1. Place a peptide aliquot ( $20 \mu \mathrm{L}, 100 \mu \mathrm{M}$ in $10 \mathrm{mM}$ MOPS, $\mathrm{pH}$ 7.4) in a circular sampling area of a 2-mm radius with a path length of $6 \mu \mathrm{m}$. The multi-reflection ATR accessory used is based on a dual-crystal technology, which has an upper silicon crystal and a hemispherical zinc selenide ( $\mathrm{ZnSe}$ ) lower crystal that does not come into contact with the sample.

2. Maintain the temperature of the sample at $20^{\circ} \mathrm{C}$

3. Collect FTIR spectra in the range of 4000 and $850 \mathrm{~cm}^{-1}$ with a resolution of $4 \mathrm{~cm}^{-1}$, a scanner velocity $20 \mathrm{kHz}, 128$ scans, a phase resolution of 32 and a zero filling factor 4 .

\subsection{Fiber diffraction of partially aligned samples}

\subsubsection{X-ray fiber diffraction}

1. Prepare XRD specimens by suspending a droplet of a peptide solution between the ends of wax-coated capillaries and allow them to dry.

2. Examine the peptides dried on the wax head forming a protuberance.

3. Compare all XRD patterns versus the XRD of the wax heads supporting the samples.

4. Mount XRD specimens vertically onto the four axis goniometer of a RAXIS IV++ X-ray diffractometer (Rigaku) equipped with a rotating anode generator.

XRD data (rings) for samples with no positional ratio between reflections correspond to average distances between objects. The average distance is the spacing calculated from the position of the ring: spacing is equal to $d=2 \times 3.14 / q$, where $q$ is a scattering vector for the position of the ring.

\subsection{Microscopy}

\subsubsection{Optical microscopy}

\subsubsection{Wide-field fluorescence microscopy}

1. Deposit a peptide aliquot $(6 \mu \mathrm{L})$ on a microscope slide and cover the slide is with a glass slip.

2. Image fluorophore-labelled fibers using an $x 40$ lens.

3. Use the DIC imaging mode to optimize the focus and record fluorescence using relevant excitation wavelengths.

4. Process the images using Carl Zeiss Vision imaging software before overlaying.

\subsubsection{Structured illumination microscopy}

1. Perform fiber imaging using a custom-built (or commercial) SIM system as described in 2.4.1.

2. Immediately prior to imaging, transfer peptide solution to a well of an 8-well chambered coverslip mounted on the microscope sample stage.

3. Capture time-lapse images under illumination of the sample with a series of nine sinusoidal irradiance patterns, with the corresponding phase gratings displayed on the SLM set such that the first diffracted orders fell inside the TIRF ring of the objective lens $(18,19)$.

4. Acquire endpoint images with a coarser grating displayed on the SLM to allow illumination of the full depth of the sample. 
5. Reconstruct the images of fibers formed at the endpoint of the self-assembly process as described in (18).

Note: Figure $4 \mathrm{~A}$ and B show a typical SIM image reconstruction of densely-packed fibers, illustrating how the improved image contrast and spatial resolution of SIM enable a clearer visualization of individual fibers against the fluorescent background. For time-lapse imaging, owing to the relatively low signal-to-noise ratio in the raw images, each sequence of raw images is summed up to create a diffraction-limited TIRF image with improved illumination uniformity and signal-to-noise.

5. Analyse time-lapse TIRF images to determine fiber growth kinetics.

6. Prior to segmentation, correct lateral offsets between images in each image series using intensity-based registration (using fluorescent clusters as markers), Figure 4C.

7. Normalize the intensity of each image and apply a top hat filter to increase image contrast.

8. Segment Individual fibers and track them through the time-lapse sequence using open active contours implemented in the ImageJ plugin JFilament (40).

Note: Values for foreground and background are defined individually for each fiber tracked in the sequence. All other contour parameters are empirically optimized once and assigned the same values for all fibers in the sequence.
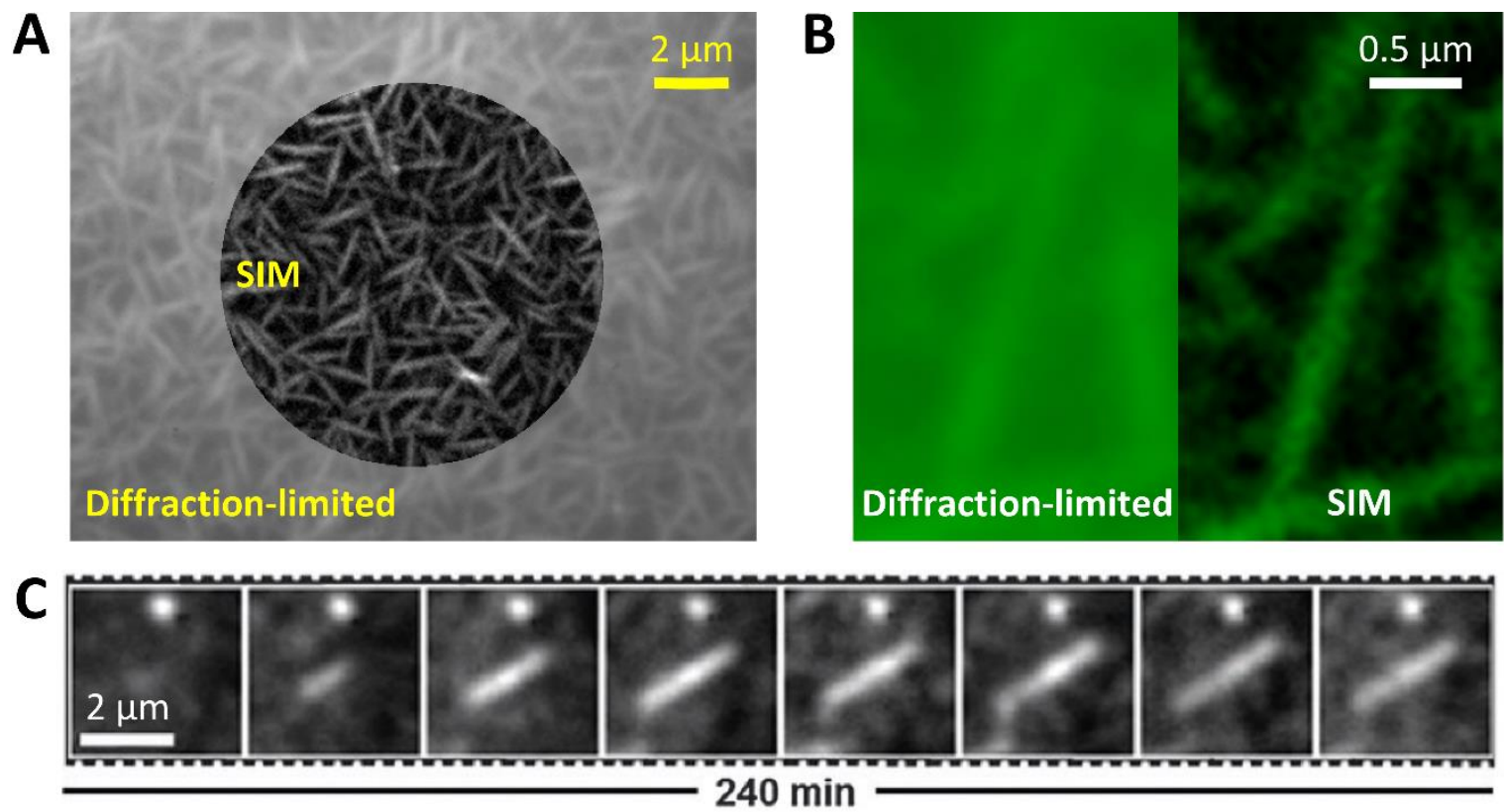

Figure 4. In situ and live imaging. (A) Composite SIM (centre circle) and diffraction-limited wide-field image (outside the circle) showing protein fibers assembled from staggered coiled-coil trimers labelled with Alexa488. (B) Montage showing diffraction-limited wide-field (left) and SIM (right) images of the same region, emphasizing how the removal of the out-of-focus light and improved spatial resolution in SIM allows clearer visualisation of individual fibers against the fluorescent background. (C) Time-lapse TIRF images of a self-assembling protein fibre. The bright round feature in the upper part of each image is a fluorescent aggregate used for image registration. Adapted by permission from Macmillan Publishers Ltd: Scientific Reports,(23) Copyright 2014.

\subsubsection{High resolution microscopy}

\subsubsection{Transmission Electron Microscopy:}

1. Record micrographs using an FEI Tecnai 20 twin lens scanning transmission electron microscope, operated at $200 \mathrm{kV}$ and equipped with an Eagle 4kx4k CCD camera. 


\subsubsection{Atomic Force Microscopy:}

1. Clean a silicon wafer using ethanol and dry the substrate under nitrogen

2. Immerge the clean silicon wafer in a solution 100-300 $\mu \mathrm{M}$ of peptide solution in MOPS at $\mathrm{pH} 7.4$ and incubated overnight at room temperature.

3. Record topographic, amplitude and phase AFM images using tapping mode AFM. All images are flattened with a first order line-wise correction fit.

4. Process Images using proprietary software (SRIP 6.0.2).

\section{References}

1. Whitesides, G.M., Boncheva, M. (2002) Beyond molecules: self-assembly of mesoscopic and macroscopic components. Proc. Natl. Acad. Sci. USA 99, 4769-4774.

2. Adler-Abramovich, L., Gazit, E. (2014) The physical properties of supramolecular peptide assemblies: from building block association to technological applications. Chem. Soc. Rev. 43, 6881-9683.

3. Whitesides, G., Mathias J., Seto C. (1991) Molecular self-assembly and nanochemistry: a chemical strategy for the synthesis of nanostructures. Science 80, 1312-1319.

4. De Santis, E., Ryadnov, M.G. (2015) Peptide self-assembly for nanomaterials: the old new kid on the block. Chem. Soc. Rev. 44, 8288-8300.

5. Hay, E. H. (2013) Cell Biology of Extracellular Matrix: Second Edition (Springer Science \& Business Media).

6. Grosse, R., Vartiainen, M.K. (2013) To be or not to be assembled: progressing into nuclear actin filaments. Nat. Rev. Mol. Cell Biol. 14, 693-697.

7. Mienaltowski, M.J., Birk, D.E. (2014) Structure, physiology, and biochemistry of collagens. Adv, Exp. Med. Biol. 802, 5-29.

8. Ross, C.A., Poirier, M.A. (2004) Protein aggregation and neurodegenerative disease. Nat. Med. 10, S10-S17.

9. Van Raaij, M.J., Mitraki, A., Lavigne, G., Cusack S. (1999) A triple beta-spiral in the adenovirus fibre shaft reveals a new structural motif for a fibrous protein. Nature 401, 935-938.

10. Papapostolou, D., Smith, A.M., Atkins, E.D.T., Oliver, S. J., Ryadnov, M.G, Serpell, L.C., Woolfson, D.N. (2007) Engineering nanoscale order into a designed protein fiber. Proc. Natl. Acad. Sci. USA 104, 10853-10858.

11. Smith, A.M., Acquah, S.F.A., Bone, N.K., Harold, W., Ryadnov, M.G., Stevens, M.S.P., Walton, D.R.M., Woolfson, D.N. (2004) Polar assembly in a designed protein fiber. Angew. Chem. Int. Ed. Engl. 44, 325-328.

12. Scheibe, I.T., Kowal, A.S., Bloom, J.D., Lindquist, S.L. (2001) Bidirectional amyloid fiber growth for a yeast prion determinant. Curr. Biol. 11, 366-369.

13. Woolfson, D.N., Ryadnov, M.G. (2006) Peptide-based fibrous biomaterials: Some things old, new and borrowed. Curr. Opin. Chem. Biol. 10, 559-567.

14. De Santis, E., Faruqui, N., Noble, J.E., Ryadnov, M.G. (2014) Exploitable length correlations in peptide nanofibres. Nanoscale 6, 11425-11430.

15. Jaroniec, C.P., MacPhee, C.E., Astrof, N.S., Dobson, C.M., Griffin, R.G. (2002) Molecular conformation of a peptide fragment of transthyretin in an amyloid fibril. Proc. Natl. Acad. Sci. USA 99, 16748-16753.

16. Rong, J., Oberbeck, F., Wang, X., Li, X., Oxsher, J., Niu, Z., Wang, Q. (2009) Tobacco mosaic virus templated synthesis of one dimensional inorganic-polymer hybrid fibres. J. Mater. Chem. 192841.

17. Poincloux, R., Lizárraga, F., Chavrier, P. (2009) Matrix invasion by tumour cells: a focus on MT1MMP trafficking to invadopodia. J. Cell. Sci. 122, 3015-3024. 
18. O'Holleran, K., Shaw, M. (2014) Optimized approaches for optical sectioning and resolution enhancement in 2D structured illumination microscopy. Biomed. Opt. Express. 2580-2590.

19. Shaw, M., Zajiczek, L., O'Holleran, K. (2015) High speed structured illumination microscopy in optically thick samples. Methods 88, 11-19.

20. Chernyatina, A.A., Hess, J.F., Guzenko, D., Voss, J.C., Strelkov, S.V. (2016) How to Study Intermediate Filaments in Atomic Detail. Meth. Enzymol. 568, 3-33.

21. Liu, C., Zhao, M., Jiang, L., Cheng, P. N., Park, J., Sawaya, M. R., Pensalfini, A., Gou, D., Berk, A.J., Glabe, C.G., Nowick J., Eisenberg D. (2012) Out-of-register $\beta$-sheets suggest a pathway to toxic amyloid aggregates. Proc. Natl. Acad. Sci. USA 109, 20913-20918.

22. Starborg, T., Lu, Y., Meadows, R.S., Kadler, K.E., Holmes, D.F., (2008) Electron microscopy in cellmatrix research. Methods. 45, 53-64.

23. Bella, A., Shaw, M., Ray, S., Ryadnov, M.G. (2014) Filming protein fibrillogenesis in real time. Sci. Rep. 4, 7529.

24. Bella, A., Ray, S., Shaw, M., Ryadnov, M. G. (2012) Arbitrary self-assembly of peptide extracellular microscopic matrices. Angew. Chem. Int. Ed. Engl. 51, 428-431.

25. Hartmann, M. D., Mendler, C. T., Bassler, J., Karamichali, I., Ridderbusch, O., Lupas, A. N., Avarez, B. H. (2016) $\alpha / \beta$ coiled coils. Elife $5,11861$.

26. Ryadnov, M.G. (2007) Peptide alpha-helices for synthetic nanostructures. Biochem. Soc. Trans. 35, 487-491.

27. Ryadnov, M.G., Bella, A., Timson, S., Woolfson, D.N. (2009) Modular design of peptide fibrillar nano- to microstructures. J. Am. Chem. Soc. 131, 13240-13241.

28. Anzini, P., Xu, C., Hughes, S., Magnotti, E., Jiang, T., Hemmingsen, L., Demeler, B., Conticello, V. P. (2013) Controlling self-assembly of a peptide-based material via metal-ion induced registry shift. J. Am. Chem. Soc. 135, 10278-10281.

29. Potekhin, S. A., Melnik, T.N., Popov, V., Lanina, N.F., Vazina ,A.A. , Rigler, P., Verdini, A.S., Corradin, G., Kajava, A.V. (2001) De novo design of fibrils made of short $\alpha$-helical coiled coil peptides. Chem. Biol. 8, 1025-1032.

30. De Santis, E., Castelletto, V., Ryadnov, M.G. (2015) Interfacial zippering-up of coiled-coil protein filaments. Phys. Chem. Chem. Phys. 17, 31055-31060.

31. Ryadnov, M.G., Woolfson, D.N. (2003) Engineering the morphology of a self-assembling protein fibre. Nat. Mater. 2, 329-332.

32. Ryadnov, M.G., Woolfson, D.N. (2005) MaP peptides: programming the self-assembly of peptidebased mesoscopic matrices. J. Am. Chem. Soc. 127, 12407-12415.

33. Faruqui, N., Bella, A., Ravi, J., Ray, S., Lamarre, B., Ryadnov, M.G. (2014) Differentially Instructive Extracellular Protein Micro-nets. J. Am. Chem. Soc. 136, 7889-7898.

34. Makin, O.S., Serpell, L.C. (2005) X-ray diffraction studies of amyloid structure. Methods Mol. Biol. 299, 67-80.

35. Kner, P., Chhun, B.B., Griffis, E.R., Winoto, L., Gustafsson, M.G. (2009) Super-resolution video microscopy of live cells by structured illumination. Nat. Methods 6, 339-342.

36. Gustafsson, M.G. (2000) Surpassing the lateral resolution limit by a factor of two using structured illumination microscopy. J. Microsc. 198, 82-87.

37. Gustafsson, M.G., Shao, L., Carlton, P.M., Wang, C.J., Golubovskaya, I.N., Cande, W.Z., Agard, D.A., Sedat, J.W. (2008) Three-dimensional resolution doubling in wide-field fluorescence microscopy by structured illumination. Biophys. J. 94, 4957-4970.

38. Pinotsi, D., Buell, A.K., Galvagnion, C., Dobson C.M., Kaminski- Schierle, G. S., Kaminski C.F. (2014) Direct observation of heterogeneous amyloid fibril growth kinetics via two-color super-resolution microscopy. Nano. Lett. 14, 339-345. 
39. Kuipers, B.J.H., Gruppen, H. (2007) Prediction of molar extinction coefficients of proteins and peptides using UV absorption of the constituent amino acids at $214 \mathrm{~nm}$ to enable quantitative reverse phase high-performance liquid chromatography-mass spectrometry analysis. J. Agric. Food. Chem. 55, 5445-5451.

40. Smith, M.B., Li, H. Shen, T., Huang, X., Yusuf, E., Vavylonis, D. (2010) Segmentation and tracking of cytoskeletal filaments using open active contours. Cytoskeleton. 67, 693-705. 